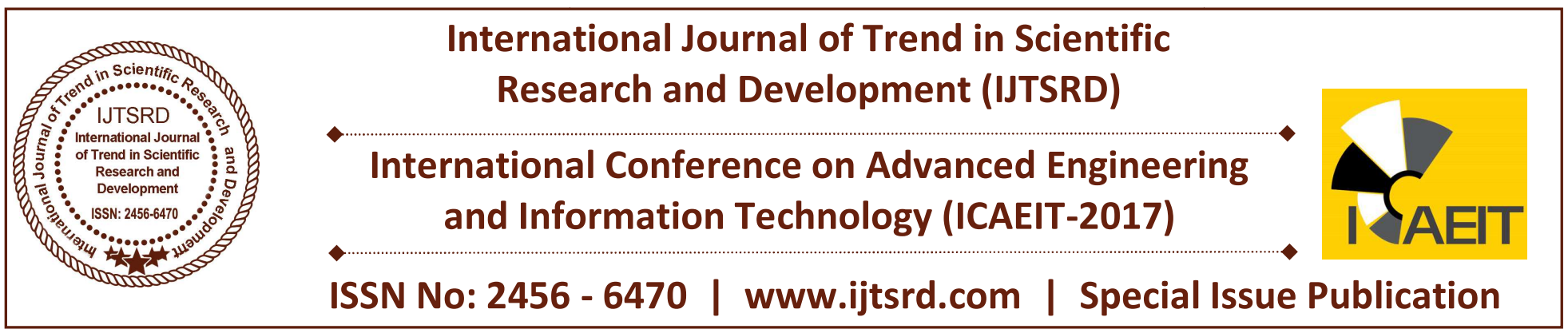

\title{
Networks Coexistence with other 2.4 GHz ISM Devices
}

\author{
Mia Torres-Dela Cruz ${ }^{1}$, Wisam Khamil Mahdloom ${ }^{2}$, Shaina Suresh ${ }^{1}$ \\ ${ }^{1}$ Faculty of Engineering and Technology, Linton University College, \\ Mantin, Negeri Sembilan, Malaysia \\ ${ }^{2}$ South Russian State Technical University, Novocherkassk, Russia
}

\section{ABSTRACT}

The possibility for the home to be networked wirelessly has greatly improved because of emerging technologies. Home networking is very much possible now with the different technologies that are available. This project is an application of three wireless technologies to machine-to-machine (M2M) home networking coexisting with other devices in the 2.4 $\mathrm{GHz}$ ISM. The networking technologies used and evaluated in the project are ZigBee, Bluetooth, and Wi-Fi, where the risk of interference in the networks is identified and evaluated. The other devices in the home are microwave oven, baby monitors, and wireless telephones.

The home network system that was developed and implemented is an interconnection of electronic home devices grouped by sub-networks categorized by each of the wireless technologies. Because the three sub networks are working in proximity, there is the high chance of interference with each other and with other devices within the $2.4 \mathrm{GHz}$ band.

The joint application of the three networks showed how these networks affect each other and how they coexist in one system and effect to and by other home devices not included in their networks but are in the same frequency range, $2.4 \mathrm{GHz}$. The paper explored the risks and characteristics of interference of these three systems with other devices when the system is implemented. Guidelines were developed to overcome and mitigate these constraints.

Keywords: Home Network, Wi-Fi, Bluetooth, microwave, Wireless Technologies, ZigBee, $2.4 \mathrm{GHz}$ ISM.

\section{INTRODUCTION}

Emerging technologies have made sure that homes have become comfortable, enjoyable, safe, and secure. Technology has been the reason so that in this era, home entertainment, comfort, safety and security are ensured and it has hugely helped to make homeowners achieve those characteristics through home networking which became popular because of Internet of Things (IoT) [21]. Managing, monitoring, and securing their homes from a remote location are now just a. They are able to operate their home devices and appliances from their jobs or while traveling. They can check what is happening in and around their homes from afar. This system is called a smart home using current and emerging network technologies where machines are able to communicate machine to machine (M2M) [1]. M2M is the course of data flow between machine to machine, and eventually machine to human, is what

$\mathrm{M} 2 \mathrm{M}$ is all about [1]. M2M is the ideal technology that is currently being used in home networking because it conserves monetary costs as well as time and effort. According to Yue, Jawen, and Rong [2], M2M networks are popularly utilized due to its low cost, power, and human intervention. The potential system that will result by using this technology is cost effective and has high service quality.

The development of home furniture and electronic devices have improved so much that the home has become more intelligent, the home has developed in terms of comfort, entertainment, security, maintenance, and management. Also because of enhanced communications and networking technologies, interconnection of these intelligent devices has resulted to a more convenient, easier and 
more efficient home networking. And because of the wireless technology the inconvenience of cabling has been replaced by wireless systems resulting to more manageable networking compared to previous systems.

The study is an investigation of the system developed where multi-technologies were used and coexisting to complement each other in a M2M home network, implemented and evaluated as to their performance regarding functionality and usability. However, there are other devices not included in the system that may interfere with the smooth operation of the system brought about by their coexisting in the same band, $2.4 \mathrm{GHz}$ ISM. Constraints such as overlap, interference, or signal issues are explored and guidelines were developed to overcome these identified constraints.

\section{Comparison of Zigbee, Bluetooth, and Wi-Fi}

The wireless technology, ZigBee, carries the IEEE standard number 802.15.4. It is categorized in the group that is involved in network operations and technologies, such as mid-sized and local networks and is wireless. Baker [3] said that the home automation standard of ZigBee is an open, wireless protocol for personal area networking (PAN) that was developed in the early 2000's as a low-cost, lowpower alternative to currently available networking protocols. Like Z-Wave, ZigBee is a "mesh networking" technology, in which packet signals are relayed by adjacent devices within the mesh until they reach their intended target. ZigBee is designed to be very tolerant to radio interference, making it a very good standard for technology-rich smart homes. Therefore, at any time and status, it is appropriate for use and development of intelligent homes [4].

The Bluetooth ${ }^{\circledR}$ technology [18] is a wireless communications technology that is simple, secure, and everywhere. Billions of devices have Bluetooth, from mobile phones, computers, and their accessories to home appliances and even medical and scientific devices. The intention is to replace the cables and wires that are connecting devices, at the same time maintaining high levels of security. The key features of Bluetooth technology are ubiquitousness, low power, and low cost. The Bluetooth specification outlines a constant configuration for an extensive range of devices to link and communicate with each other.

Bluetooth technology is convenient when transmitting information amongst two or more devices that are nearby each other when rapidity is not an issue. Devices such as telephones, printers, modems and headsets are suitable for this connectivity. It is best appropriate to low-bandwidth applications such as transferring sound data through telephones, i.e., with a Bluetooth headset or speakers, or byte data with handheld devices such as tablets for transferring files or for mouse and keyboard.

Wi-Fi is the popular name for wireless local area networks which is based on the IEEE $802.11 \mathrm{~b}$ standard is Wi-Fi [5]. This technology is considered one of the most brilliant areas of the information and communications business [6]. Wi-Fi has taken broadbased interest all over technical and popular areas. It is progressively being viewed as the medium that will bring in several types of technologies that would support or supported by Internet access to the general population [7].

Most Wi-Fi devices in the home network are power intensive. Although the network itself is wireless, the devices are connected to outlets and use a lot of power. For operating networks that are full-scale, Wi$\mathrm{Fi}$ is considered a better choice because faster connection is enabled, base station range is better, and, if configured correctly, has better security.

According to Pothuganti and Chitneni [8], obviously, Wi-Fi provides a higher data rate, compared to Zigbee and Bluetooth. Zigbee is well suited for WPAN communication (about $10 \mathrm{~m}$ ), Bluetooth is suited to PAN, while Wi-Fi is oriented to WLAN (about $100 \mathrm{~m})$.

Tables 1 and 2 below showed the performance of each of the technologies using the criteria specified for both functionality and usability. The following were measured during testing of the system [9]:

Table 1: Comparative functionalities of Bluetooth, Zigbee and Wi-Fi

\begin{tabular}{|c|c|c|c|}
\hline Criteria & Bluetooth & Zigbee & Wi-Fi \\
\hline Application Focus & $\begin{array}{l}\text { Personal Area } \\
\text { Network } \\
\text { (PAN), Mobile } \\
\text { device control, } \\
\text { i.e. phone, } \\
\text { keypad, etc. }\end{array}$ & $\begin{array}{l}\text { Electricity } \\
\text { switching } \\
\text { control, i.e. } \\
\text { light bulb, } \\
\text { representatio } \\
\text { n of home } \\
\text { electronic } \\
\text { devices }\end{array}$ & $\begin{array}{l}\text { Home } \\
\text { monitoring, } \\
\text { Video and } \\
\text { Photo by } \\
\text { remote } \\
\text { control }\end{array}$ \\
\hline $\begin{array}{l}\text { Transmission } \\
\text { Range (meters) }\end{array}$ & 10 & 30 & $1-100$ \\
\hline Success Matrix & $\begin{array}{l}\text { Cost } \\
\text { convenience }\end{array}$ & $\begin{array}{l}\text { Power, } \\
\text { reliability }\end{array}$ & $\begin{array}{l}\text { Speed, } \\
\text { Flexibility, } \\
\text { Portability }\end{array}$ \\
\hline Data Protection & 16 bit $\mathrm{CRC}$ & 16 bit $\mathrm{CRC}$ & 32 bit CRC \\
\hline Optimized for & $\begin{array}{l}\text { Low cost, } \\
\text { convenience }\end{array}$ & $\begin{array}{l}\text { Low cost, } \\
\text { low power }\end{array}$ & $\begin{array}{l}\text { Speed, } \\
\text { security }\end{array}$ \\
\hline Frequency & $2.4 \mathrm{GHz}$ & $\begin{array}{l}900 \mathrm{MHz} \text { to } \\
2.4 \mathrm{GHz}\end{array}$ & $\begin{array}{l}2.4 \text { and } 5 \\
\mathrm{GHz}\end{array}$ \\
\hline
\end{tabular}


Table 2: Comparative usabilities of Wi-Fi and ZigBee

\begin{tabular}{l|l|l|l}
\hline \hline Criteria & Bluetooth & Zigbee & Wi-Fi \\
\hline $\begin{array}{l}\text { Power } \\
\text { Consumption } \\
\text { (days) }\end{array}$ & Low & Very low & High \\
\hline System Resource & $25 \mathrm{~KB}$ & $4-32 \mathrm{~KB}$ & $1 \mathrm{MB}$ \\
\hline $\begin{array}{l}\text { Battery life } \\
\text { (Days) }\end{array}$ & $1-7$ & $100-1000+$ & 5 \\
\hline Bandwidth & $720 \mathrm{~KB} / \mathrm{S}$ & $\begin{array}{l}20-250 \\
\mathrm{~KB} / \mathrm{S}\end{array}$ & $11000 \mathrm{~KB} / \mathrm{S}$ \\
\hline Data Rate & $1 \mathrm{Mbits} / \mathrm{s}$ & $115.2 \mathrm{Kbits} / \mathrm{s}$ & $\begin{array}{l}11 \text { and } 54 \\
\text { Mbits/s }\end{array}$ \\
\hline Reliability & Low & High & Medium \\
\hline \hline
\end{tabular}

Wi-Fi, Bluetooth, and ZigBee networks share the same frequency band which is $2.4 \mathrm{GHz}$. Because of this, these technologies usually operate in proximity and the system developed requires that they have to co-exist together. However, WiFi consumes higher power level, while Bluetooth and ZigBee use low power [10].

\section{The $2.4 \mathrm{GHz}$ ISM Band}

ISM band means Industrial, Scientific and Medical band. This is a part of the radio spectrum that can be used for any purpose without a license in most countries. The 902-928 MHz, $2.4 \mathrm{GHz}$ and 5.7-5.8 $\mathrm{GHz}$ bands were initially used in the United States of America for machines that emitted radio frequencies, i.e., RF welders, industrial heaters, and microwave ovens. In 1985, the FCC Rules (Part 15.247) opened up the ISM bands for wireless LANs and mobile communications. In 1997, it added additional bands in the $5 \mathrm{GHz}$ range under Part 15.407, known as the Unlicensed National Information Infrastructure (UNII) [19].

A lot of applications use the ISM/U-NII bands, including cordless phones, wireless garage door openers, wireless microphones, vehicle tracking and amateur radio [19].

$2.4 \mathrm{GHz}$ band is one of the most popular bands 2.4 $\mathrm{GHz}$ in ISM. ISM bands are unlicensed, which makes it easier to certify the equipment with FCC (or its counterparts in other countries). Among all the other ISM bands, $2.4 \mathrm{GHz}$ is an international band. These devices may use standard protocols such as the IEEE $802.11 \mathrm{~b} / \mathrm{g} / \mathrm{n}$, or they may use proprietary protocols [20]. The radios may use frequency hopping, frequency agility, or may operate on a fixed frequency. Because of these reasons, a lot of wireless devices operate on $2.4 \mathrm{GHz}$ band. The following are some of the most popular ones, Wi-Fi, Bluetooth, ZigBee, microwave ovens, cordless phones, wireless microphone, baby monitors, car alarms, and RC toys.

These various devices may coexist one time or the other and the problem is interference may occur between them.

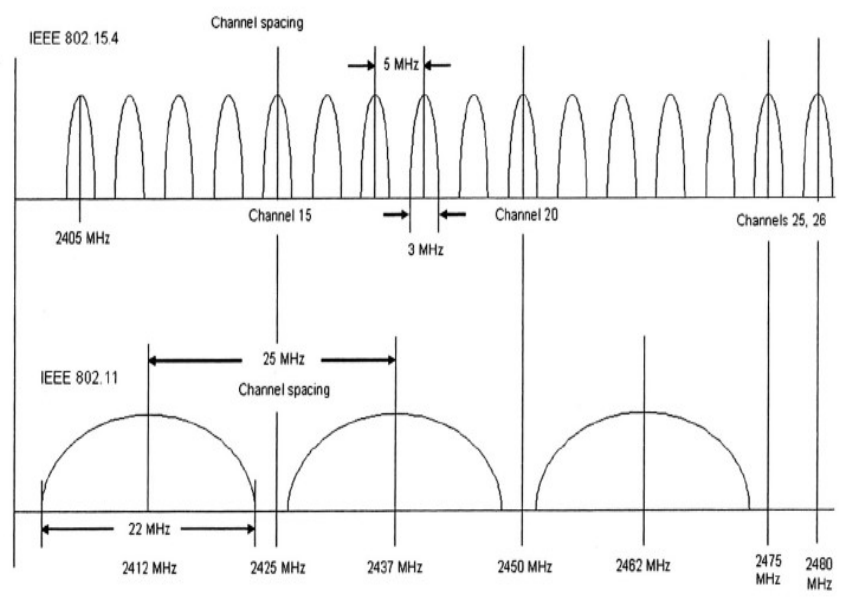

Figure 1: ZigBee (802.15.4) and Wi-Fi (802.11) Channels (Source: Mobiusconsulting.com)

Arif and Supankat [11] stated that interference will occur on networks due to the large number of devices and several different wireless communication technologies are connected. Because of this interference, a decrease in the performance of the wireless networks is noted. Wi-Fi and ZigBee are expected to run simultaneously in close proximity due to the demand for ubiquitous Internet access and increasingly, these two networks are being utilized in urban areas to support real-time and long-term monitoring [10]. Interests in using these two networks to coexist in different systems have intensified and one of these systems is in smart homes. However, because of the overlap in the channels of ZigBee and $\mathrm{Wi}-\mathrm{Fi}$, there is a cause for concern.

Yan, et al [14] stated that ZigBee is vulnerable to WiFi for two reasons, one, the transmission power of Wi-Fi is approximately 10 to 100 times higher than ZigBee which means it is probably affected by the high power of the Wi-Fi devices; and two, because ZigBee has low data rate and cost, it is inferred that the ability for time resolution becomes slow and unstable. In this research by Yan et. al., they developed a single-antenna sink based design which coexisted with ZigBee and Wi-Fi, called Wizbee. This 
was developed to apply interference cancelation technique in order to mitigate interference from $\mathrm{WiFi}$, and to extract signals from ZigBee.

A series of experiments by Zhao et al [15] resulted in the findings concerning symmetric and asymmetric scenarios in Zigbee-Wi-Fi interference. Accordingly, in symmetric scenarios, ZigBee's throughput degrades heavier than that of $\mathrm{Wi}-\mathrm{Fi}$ and the packet losses is mostly because of ACF. While in asymmetric scenarios, there's a difficulty for ZigBee (802.15.4) to persist because of the interference of Wi-Fi (802.11n), since its Packet Delivery Ratio (PDR) is approaching zero. The reason for the packet losses are because of auto-correlation function (ACF) and corruption.

A simulation by Arif and Supangkat [11] was more related to the system developed in this study because of the similarities of connections and usage of the topologies. Their simulation was done by comparing the throughput of the system by measuring the wireless performance in two ways, one, with only ZigBee and two, with both ZigBee and Wi-Fi simultaneously, in order to find the impact of interference. On the first scenario (ZigBee only), throughput is above $35 \mathrm{Kbps}$. But in the second scenario when Wi-Fi was introduced, the throughput of ZigBee significantly dropped below $35 \mathrm{Kbps}$. From this, the authors inferred that the introduction of WiFi working simultaneously with ZigBee degraded transmission performance of the latter.

Extensive experiments were done by $\mathrm{Li}$ et al [16] to demonstrate their proposal in tackling issues of the co-existence of ZigBee and Wi-Fi, which are to shift the paradigm from traditional discrete channel allocation to continuous frequency allocation; innovate in transforming frequency allocation into spatial tessellation in a frequency-distance space, and propose an algorithm to compute a near optimal solution in a localized manner. The authors designed a probabilistic mechanism to adaptively use CSMA according to real-time interference assessments; it can fine-tune the tradeoff between transmission throughput and quality.

\section{Findings}

In the paper of Madhloom et al. [21], findings show that positive interference were identified in the set up for the system, issues were detected when the sub networks were run simultaneously. Three set ups were done to observe the signals of the system. These were:
Wi-Fi alone, ZigBee alone, and Wi-Fi and ZigBee together.

Wi-Fi alone. This is the normal situation where the performance of the system with only Wi-Fi running is noted. In this set-up, all three IP cameras were launched, initially one at a time and then two, then all three at the same time. Performance of the Wi-Fi was noted and inferred as normal.

ZigBee alone. This situation also is the basis for the comparison. ZigBee sub network was launched alone and three representative devices were launched, initially one at a time, then two, then finally all three together.

Wi-Fi and ZigBee together. The two networks would be tested for its effect with each other. Both will be deployed simultaneously and will be observed for any difference as compared with running alone.

The result of the evaluation in the Wi-Fi and ZigBee is a positive interference for ZigBee throughput. The signal deteriorated and at some point, the relay for one device did not work. However, Wi-Fi signal has very little change when run simultaneous with ZigBee, with value of change as negligible, as compared with signal when running alone. This corroborates the contention of Arif and Supangkat [11] who did a simulation for ZigBee and Wi-Fi coexistence and measured the throughput for ZigBee alone and then ZigBee together with Wi-Fi. For ZigBee alone, the throughput reached more than $35 \mathrm{Kbps}$ but deteriorated to above $30 \mathrm{Kbps}$ when launched with Wi-Fi (Figure 5).

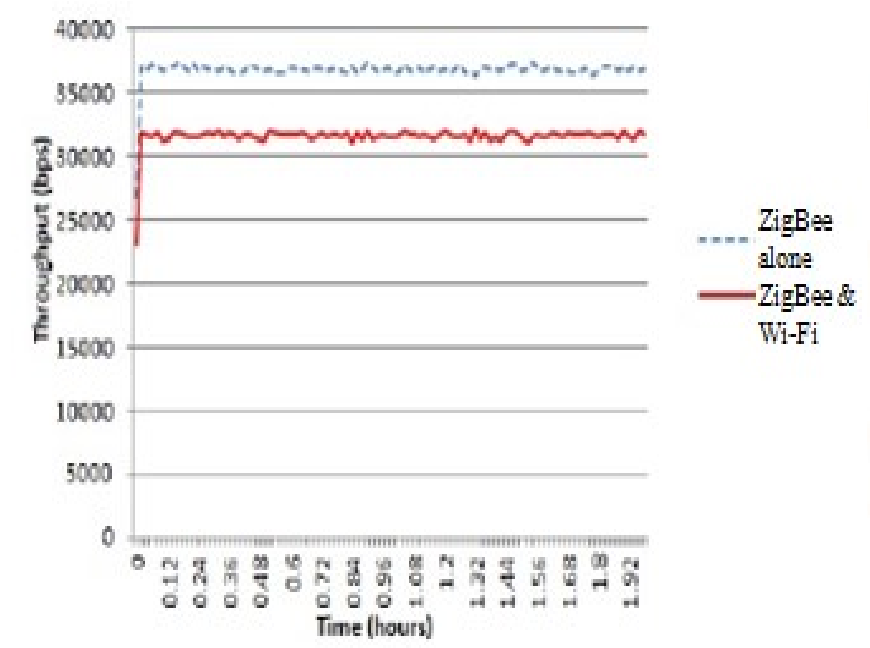

Figure 2: Throughput of ZigBee Alone and ZigBee and Wi-Fi Together (Source: [11]) 


\section{Mitigating Solutions for avoiding interference among devices in $2.4 \mathrm{GHz}$ band}

One solution to avoid interference is to replace Zigbee with a similar low power network such as WizBee. WizBee [14] is designed to be compatible with current ZigBee and WiFi system, and do not need any protocol modification. The requirement is to replace conventional ZigBee sink with WizBee sink.

On situations where there is interference, Multi Header $(\mathrm{MH})$ solution may be applied. It is effective when there is a loss of ZigBee packet, which is because of Header error. Header error is a result of Wi-Fi interference with ZigBee. MH scheme [17] is used to reduce the effective throughput which determines the number of headers adaptively based on the presence of heterogeneous networks. This solution is only effective when interference is present, which allows better throughput and efficiency.

\section{Conclusion}

M2M home networking using the three technologies, Wi-Fi, Bluetooth and ZigBee provide convenience of connection and communication within the system. However, there are issues with their coexistence with other devices operating in the same band, $2.4 \mathrm{GHz}$, in the home because of their different characteristics.

The project resulted to a well-functioning M2M home network composed of joint wireless technologies, ZigBee, Bluetooth and Wi-Fi networks. The coexisting technologies were observed, compared, and documented according to their functionalities and usabilities (tables 1 and 2) in the specific system itself.

The issues of interference between the three network technologies were observed during the test run of the system. It was perceived that when all are deployed at the same time, ZigBee signal deteriorates which makes some devices lose relay from the network. However, when working alone, the signal for ZigBee is of normal rate. Wi-Fi does not seem to get affected by ZigBee and Bluetooth at all.

Despite the interference, it is believed that ZigBee, Bluetooth and Wi-Fi can coexist together when it can be mitigated through good set-up planning. Solutions are available to avoid or mitigate issues of interference because of ZigBee, Bluetooth and Wi-Fi coexistence.
For future work, it is recommended that new systems involving co-existence with ZigBee and Wi-Fi should plan set-up very well to consider new standards which will favor different channels and frequencies to avoid clashing.

Further studies for other network interference should be done such as ZigBee interference with BlueTooth, ZWave, and other technologies such as Li-Fi is recommended.

\section{REFERENCES}

1. W. Madhloom and M. Dela Cruz (2015), "M2M Home Network Using 3 Selected Wireless Technologies",

http://www.ijetae.com/files/ICSMET15/IJETAE_I CSMET_2015_03.pdf

2. L. Yue, K. Jiawen, and Y. Rong, (2013), "Efficient and Secure Resource Management in Home M2M Networks", International Journal of Distributed Sensor Networks, October 2013.

3. D. Baker, August 16, 2013, How ZigBee Compares in Wireless Home Automation. https://www.control4.com/blog/2013/08/howzigbee- compares-in-wireless-home-automation. [Accessed: 15- Sept- 2017].

4. F. Anan, X. Xiaoling, Y. Wenling, Z. Li, "The Realization of Intelligent Home by ZigBee Wireless Network Technology," Circuits, Communications and Systems, 2009. PACCS '09. Pacific-Asia Conference on , vol., no., pp.81,84, 16-17 May 2009.

5. C. Cordeiro, D. Akhmetov, and M. Park, (2010). IEEE 802.11 ad: introduction and performance evaluation of the first multi-gbps Wi-Fi technology. pp.3--8.

6. "Connect Your Life | Wi-Fi Alliance", Wi-fi.org, 2017. [Online]. Available: https://www.wifi.org/discover-wi-fi/connect-your-life. [Accessed: 15- Sept- 2017].

7. B. Mitchell, "How wireless and computer networks help you do work and have more fun", Lifewire, 07 June 2017. [Online]. Available: https://www.lifewire.com/benefits-of-wirelesshome-computer-networking-816541. [Accessed: 06- Sept- 2017].

8. K. Pothuganti, and A. Chitneni, "A comparative study of wireless protocols: Bluetooth, UWB, Zigbee, and Wi-Fi”, Advance in Electronic and 
Electric Engineering, ISSN 2231-1297, vol. 4, no. 6 (2014), pp. 655-662.

9. W. Madhloom, and M. Dela Cruz, "Comparative M2M Home Network Application of Bluetooth, Zigbee, and Wi-Fi”, IACITE 2015, January 2015.

10. S. Jacob, P. Ravi, "Enabling Coexistence of ZigBee and WiFi", Communications on Applied Electronics (CAE) - ISSN : 2394-4714, New York, USA,Volume 2 - No.6, August 2015 www.caeaccess.org

11. S. Arif and S. Supangkat, "Simulation and Analysis of ZigBee - WiFi Interference”, ICT For Smart Society (ICISS), 2014 International Conference, IEEE Explore, 24-25 Sept. 2014.

12. Crossbow, 2017. "Avoiding $R F$ Interference Between WiFi and Zigbee" [Online]. Available: https://www.mobiusconsulting.com/ papers/ZigBeeandWiFiInterference.pdf. [Accessed: 08- Oct- 2017].

13. "Wi-Fi RF Spectrum; interferences; how to detect them, case study: microwaves. | Acrylic WiFi", Acrylic WiFi, 2017. [Online]. Available: https://www.acrylicwifi.com/en/blog/wifi-rfspectrum-detect-study-case-microwave/. [Accessed: 08- Oct- 2017].

14. Y. Yan, P. Yang, X. Y. Li, Y. Zhang, J. Lu, L. You, J. Wang, J. Han, Y. Xiong. (2015). “WizBee: Wise ZigBee Coexistence via Interference Cancellation with Single Antenna," in IEEE Transactions on Mobile Computing, vol. 14, no. 12, pp. 2590-2603, Dec. 12015. doi: 10.1109/TMC.2014.2359673
15. Z. Zhao, X. Wu, X. Lai, J. Zhao, X. Li, “ZigBee vs WiFi: Understanding issues and measuring performances of their coexistence", Performance Computing and Communications Conference (IPCCC), 2014 IEEE International, 5-7 Dec. 2014, Austin, TX, USA.

16. F. Li, J. Luo, G. Shi, Y. He, "ART: Adaptive fRequency-Temporal Co-Existing of ZigBee and WiFi", IEEE Transactions on Mobile Computing, Vol. 16, No. 3, March 2017

17. P. D. Sai Manoj, G. V. K. Sasirekha, and J. Bapat, "A Novel Approach for Coexistence of ZigBee with WiFi," International Journal of Computer and Communication Engineering vol. 1, no. 3, pp. 251-269, 2012.

18. Bluetooth.com. The Global Standard for Connection. [Online] Available at https://www.bluetooth.com/bluetooth-technology. Accessed: 03 December 2017.

19. PCMag.com, (2017) ISM Band. [Online] Available

at:https://www.pcmag.com/encyclopedia/term/454 67/ism-band. Accessed on: 03 December 2017.

20. Intel.com, (2012) USB 3.0* Radio Frequency Interference Impact on $2.4 \mathrm{GHz}$ Wireless Devices. Paper Document: 327216-001. [Online] Available at http://www.intel.com/design/literature.htm. Accessed on: 13 December 2017.

21. Madhloom W., Dela Cruz M., Kayalvizhi S (2017) Coexistence of ZigBee and Wi-Fi Networks in Machine-to-Machine Home System, ICAEIT 2017, Linton University College, Mantin, Malaysia. 